\title{
Correlated Wide Scale Imaging of Tissue Sections on Glass Slides for Histology and Ultrastructural Pathology by Backscatter Electron Scanning Microscopy
}

\author{
Mike Reichelt $^{1}$, Meredith Sagolla ${ }^{1}$, Cary Austin ${ }^{1}$, Patrick Caplazi ${ }^{1}$ and Joshua Webster ${ }^{1}$ \\ 1. Department of Pathology, Genentech, South San Francisco, USA.
}

Electron microscopy (EM) of tissues continues to play an important role in pathology, diagnostics, safety assessment and basic biomedical research. Analysis of tissues at the ultrastructural level is most often performed with the transmission electron microscope (TEM). Traditionally, sample blocks processed for TEM are first analyzed by preparing thick sections stained with Toluidine-Blue on glass slides for light microscopy (LM) evaluation of processing quality, characterization of the histopathology, and to identify a region of interest (ROI) for ultrastructural evaluation. After a ROI has been found, sample blocks need to be further trimmed and reevaluated by LM before ultrathin sections $(50-100 \mathrm{~nm})$ are collected on small grids and stained for analysis by TEM.

This procedure has been used for decades with great success. Nevertheless, it involves major challenges and technical hurdles: 1.) Preparation of ultrathin sections is time consuming and requires skilled personnel. 2.) Despite careful preparation ultrathin sections frequently contain areas with contamination, folds or knife marks which can obscure important details. 3.) TEM grids are small and limit the size of a tissue section that can be analyzed; in addition, grid bars can further obscure the field of view. 4.) TEM grids are fragile and require carful handling and storage. 5.) Correlation of a ROI between LM and TEM can be extremely challenging and time consuming, especially with large and complex tissues (e.g. brain) or in homogenous samples (e.g. cell culture) with no "landmarks". 6.) Switching from LM to TEM analysis requires parallel sectioning; therefore, the ROI analyzed by TEM is not exactly the same area that was identified in the LM.

Imaging of cell and tissue sections directly on glass slides with backscatter electron (BSE) scanning electron microscopy (SEM) promises immense potential for the field of ultrastructural pathology and can overcome all of the challenges mentioned above [1]. BSE imaging exploits the fact that atoms of different atomic weight differ in their backscatter coefficient: the higher the atomic number (z), the more electrons are scattered back. This can be exploited for z-contrast imaging of biological samples with dedicated BSE detectors. Numerous protocols have been developed for BSE-SEM imaging, especially for nervous tissue, that maximize the staining of proteins, nucleic acids and lipids with heavy metals like uranium, lead and osmium [2].

Surprisingly, in the field of ultrastructural pathology, BSE imaging of tissues has hardly been reported. We therefore evaluated and modified existing processing protocols specifically for the analysis of tissues that are most relevant in the field of ultrastructural pathology, including healthy and diseased kidney, lung, intestine, skeletal muscle, heart muscle and brain tissues. We used these optimized processing protocols in conjunction with imaging protocols that make full use of the large store resolution (32k x 24k) and improved detectors found in modern field emission SEMs.

For BSE imaging we started with the "in chamber" dedicated backscatter electron detector (BSD) for wide field overview imaging of tissues at low magnifications $(50 \mathrm{x}$ to $500 \mathrm{x})$ to find tissue sections, characterize the histology and define ROIs. We then continued using the BSD1 detector at higher 
magnifications, or, if the maximum resolution was required, we switched to the more sensitive "inlens" energy selective backscatter detector (ESB) that can be efficiently operated at lower acceleration voltages $(1.5 \mathrm{kV})$. This workflow allowed us to analyze the same tissue section at the histology level before "zooming in" to analyze ROIs at the ultrastructural level without needing to switch microscopes or preparing parallel ultrathin sections. This saves significant "hands on" time and, most importantly, allows for full correlation of the ultrastructural detail with the histological context.

An example is shown in Figure 1. Kidney tissue was prepared with a protocol that used reduced osmium for membrane contrast and tannic acid as a mordant to increase the staining of protein and nucleic acids with uranyl acetate and lead aspartate. Tissue sections (1 micron thick) were placed on carbon-coated standard glass slides, post stained and then imaged with a Zeiss GeminiSEM300. The BSD1 detector was used at $2.5 \mathrm{kV}$ with $100 \mathrm{x}$ to $500 \mathrm{x}$ magnifications to find the tissue sections, histologically evaluate the kidney, and identify a glomerulus (top panel). At magnification up to 5000x the glomerulus was explored to find relevant capillary and urinary spaces (middle panel). Finally, ultrastructural detail important for renal pathology including the basement membrane, podocyte foot processes and capillary fenestrations were imaged with magnifications up to 40000x (bottom panel).

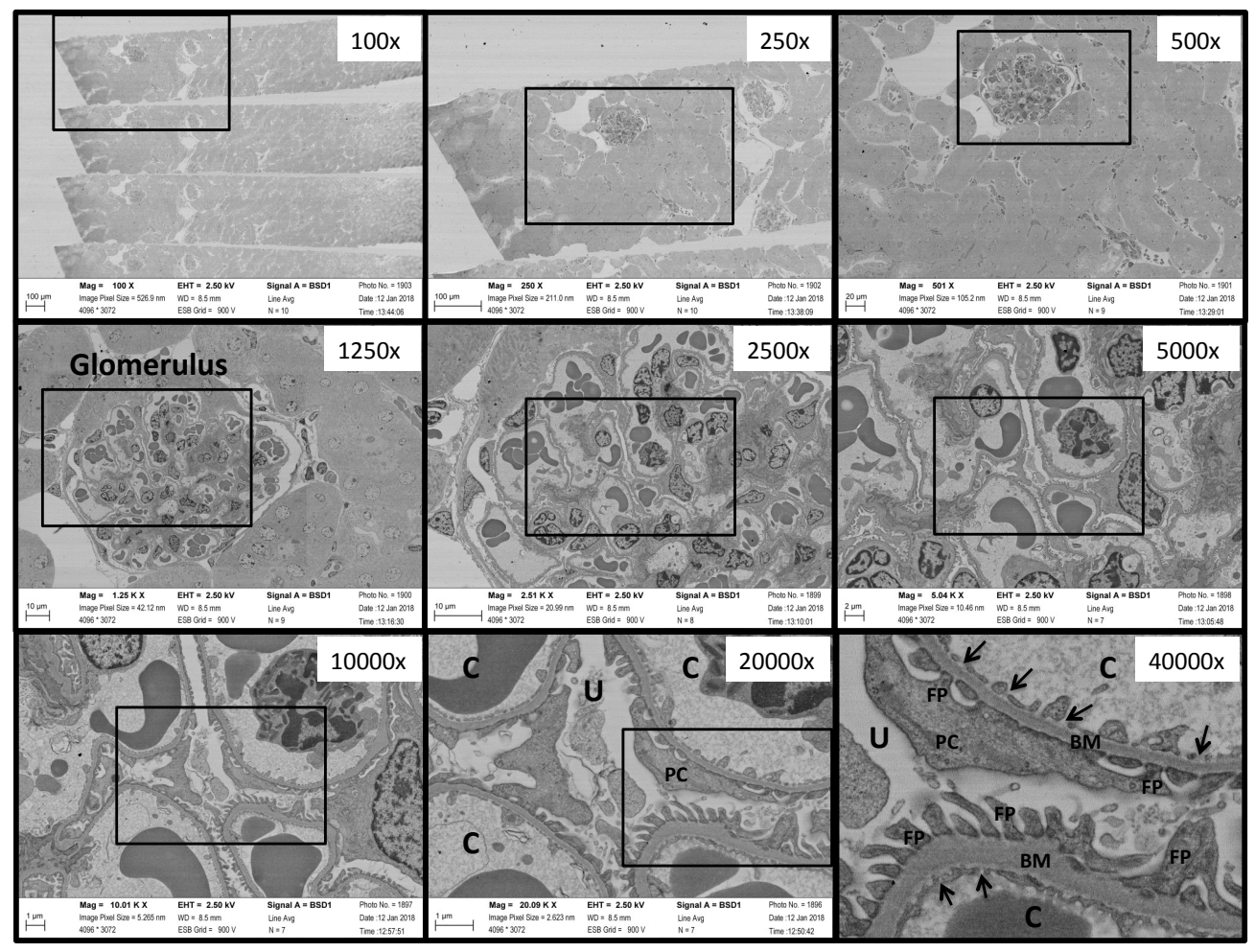

Figure 1. Correlated wide scale analysis of a kidney glomerulus. Sections of kidney tissue processed for BSE imaging were placed on carbon-coated standard glass slides. Imaging was performed with a Zeiss GeminiSEM300 at magnification from 100x to 40000x. Black squares indicate the field of view of the next higher magnification in the panel. Capillary space $(\mathrm{C})$, urinary space $(\mathrm{U})$, basement membrane $(\mathrm{BM})$, podocytes $(\mathrm{PC})$, podocyte foot processes (FP) and capillary fenestrations (arrows) are marked.

[1] M Reichelt et al., PLoS Pathogens 8 (2012), e 1002740

[2] JC Tapia et al., Nature Protocols 7 (2012), p.193 